\title{
A Study on Ricci Dark Energy in Bulk-Brane Interaction
}

\author{
Surajit Chattopadhyay \\ Pailan College of Management and Technology, Bengal Pailan Park, Kolkata 700 104, India \\ Correspondence should be addressed to Surajit Chattopadhyay; surajitchatto@gmail.com
}

Received 16 July 2013; Accepted 19 August 2013

Academic Editors: A. Koshelev, L. Marek-Crnjac, and I. V. Vancea

Copyright (C) 2013 Surajit Chattopadhyay. This is an open access article distributed under the Creative Commons Attribution License, which permits unrestricted use, distribution, and reproduction in any medium, provided the original work is properly cited.

\begin{abstract}
We have investigated the effects of the interaction between a brane universe and the bulk in which it is embedded. Considering the effects of the interaction between a brane universe and the bulk, we have obtained the equation of state for the interacting holographic Ricci dark energy density $\rho_{\Lambda}=3 c^{2}\left(\dot{H}+2 H^{2}\right)$ in the flat universe. We have investigated the impact of $c^{2}$ on the equation of state $\omega_{\Lambda}$. Also, considering the power law for of the scale factor, we have observed that nontrivial contributions of dark energy which differ from the standard matter fields confined to the brane are increasing with the evolution of the universe.
\end{abstract}

\section{Introduction}

The observed accelerated expansion of the universe is usually attributed to the presence of an exotic kind of energy, called "dark energy" (DE) [1-8]. The measurement of WMAP and the supernova analysis predicts that exactly $95.4915028 \%$ of the cosmos must be dark energy [9-11]. A great variety of DE models have been proposed so far. Models of dark energy are conveniently characterized by the equation of state parameter $\omega=p / \rho$, where $\rho$ is the energy density and $p$ is the pressure [12-15]. The models are discussed at length in the references mentioned above. Examples of DE models are tachyon [16], phantom [17], Chaplygin gas [18], K-essence [19], hessence [20], dilaton [21], holographic dark energy [22], and so forth. Observational evidence in support of DE is SNIa, CMB, and BAO [23-25]. The Type Ia supernova (SNIa) observations, cosmic microwave background (CMB), and baryon acoustic oscillations (BAO) have confirmed that about $73 \%$ of the energy density of the present universe consists of dark energy. The so-called "holographic dark energy" (HDE), based on the holographic principle [26], was proposed by Li [27]. In holographic principle the black holes are regarded as the maximally entropic objects of a given region and this principle postulates that the maximum entropy inside this region behaves nonextensively, growing only as its surface area [28]. Therefore, the number of independent degrees of freedom is bounded by the surface area in Planck units.
This implies that an effective field theory with UV cutoff $\Lambda$ in a box of size $L$ is not self-consistent, if it does not satisfy the Bekenstein entropy bound [28]. It was suggested in [29] that the total energy in a region of size $L$ should not exceed the mass of a black hole of the same size. Based on this assumption, [27] proposed HDE density as

$$
\rho_{h}=3 c^{2} M_{p}^{2} L^{-2},
$$

where $c^{2}$ is a dimensionless constant. Hsu [30] took Hubble horizon as the IR cutoff in HDE, and subsequently the HDE is written as $\rho_{h}=3 c^{2} M_{p}^{2} H^{2}$. This consideration by [30] failed to give the accelerated expansion of the universe. Gao et al. [31] proposed a holographic dark energy model in which the future event horizon is replaced by the inverse of the Ricci scalar curvature and they [31] dubbed this model as the Ricci dark energy model (RDE). The Ricci curvature of FRW universe is given by

$$
R=-6\left(\dot{H}+2 H^{2}+\frac{k}{a^{2}}\right) .
$$

The density of the RDE is therefore [32]

$$
\rho_{\Lambda}=3 c^{2} M_{p}^{2} R=3 c^{2} M_{p}^{2}\left(\dot{H}+2 H^{2}+\frac{k}{a^{2}}\right) .
$$


Cai et al. [33] pointed out that the Ricci dark energy can be viewed as originating from taking the causal connection scale as the IR cutoff in the holographic setting. Here we mention some significant works on RDE. Feng [34] investigated the statefinder diagnostics of the RDE. Feng [35] investigated the nature of $\mathrm{RDE}$ with respect to the holographic principle of quantum gravity theory. Feng [36] regarded the $f(R)$ theory as an effective description for the acceleration of the universe and reconstruct the function $f(R)$ from the RDE. Feng and $\mathrm{Li}$ [37] investigated the viscous RDE model by assuming that there is bulk viscosity in the linear barotropic fluid and the RDE. In another work Feng and Zhang [38] discussed the role of $c^{2}$ in the RDE density (3) and revealed that if $c^{2}<0.5$, then equation of state of RDE will evolve across the cosmological constant boundary -1 , that is, RDE will behave like quintom. In the present work, we are going to discuss the RDE in bulkbrane interaction.

In recent years, the idea that the universe is a brane embedded in a higher-dimensional space and has got much attraction of the researchers [39-46]. Friedmann equation on the brane contains corrections of the usual four-dimensional equation [42]. Binetruy et al. [41] found a term $H \propto \rho$, which is observationally problematic. The model is consistent if a cosmological constant in the bulk and the tension on the brane are considered. This leads to a cosmological version of the Randall-Sundrum scenario of warped geometries [42]. van de Bruck et al. [42] considered an interaction between the bulk and the brane, which is another nontrivial aspect of brane world theories. The purpose of the present work is to disclose the effect of the energy exchange between the brane and the bulk on the evolution of the universe by considering the flow of energy onto or away from the brane. Employing the RDE in a flat universe, we obtain the equation of state parameter for RDE density. This study is motivated by the works of [47-50]. In an interaction between the bulk and the brane, [47] considered holographic model of dark energy in nonflat universe under the assumption that the CDM energy density on the brane is conserved, but the holographic dark energy density on the brane is not conserved due to brane-bulk energy exchange. Sheykhi [48] considered the agegraphic models of dark energy in a braneworld scenario with brane-bulk energy exchange under the assumption that the adiabatic equation for the dark matter is satisfied while it is violated for the agegraphic dark energy due to the energy exchange between the brane and the bulk. In the study of [48], the author revealed the equation of state (EoS) parameter can evolve from the quintessence to phantom. Myung and Kim [49] introduced the brane-bulk interaction to discuss a limitation of the cosmological Cardy-Verlinde formula which is useful for the holographic description of brane cosmology and showed that in the presence of the branebulk interaction, one cannot find the entropy representation of the first Friedmann equation. Saridakis [46] considered a generalized version of holographic dark energy arguing that it must be considered in the maximally subspace of a cosmological model and showed that in the context of brane cosmology it leads to a bulk holographic dark energy which transfers its holographic nature to the effective 4D dark energy. Saridakis [51] applied the bulk holographic dark energy in general 5D two-brane models and extracted the Friedmann equation on the physical brane and showed that in the general moving brane case the effective 4D holographic dark energy behaves as a quintom for a large parameter-space area of a simple solution subclass.

In the present study we consider an interaction between the bulk and the brane, which is a nontrivial aspect of braneworld theories. We will discuss the flow of energy onto or away from the brane universe. Then, using the holographic $\mathrm{RDE}$ in flat universe obtained equation of state for interacting RDE. Firstly, we review the formalism of bulk-brane energy exchange. Then we apply this material to a brane-world cosmology under the assumption that the cold dark matter energy density on the brane is conserved, but the RDE density on the brane is not conserved due to brane-bulk energy exchange.

\section{Bulk-Brane Energy Exchange}

The bulk-brane action is given by $[47,52]$ :

$$
S=\int d^{5} x \sqrt{-G}\left(\frac{R_{5}}{2 \kappa_{5}^{2}-\Lambda_{5}+L_{B}^{m}}\right)+\int d^{4} x \sqrt{-g}\left(-\sigma+L_{b}^{m}\right),
$$

where $R_{5}$ is the curvature scalar of the five-dimensional metric, $\Lambda_{5}$ is the bulk cosmological constant, $\sigma$ is the brane tension, and $L_{B}^{m}$ and $L_{b}^{m}$ are the matter Lagrangian in the bulk and on the brane, respectively. We consider the cosmological solution with a metric of the form $[47,52]$ :

$$
\begin{aligned}
d s^{2}= & -n^{2}(t, y) d t^{2}+a^{2}(t, y) \gamma_{i j} d x^{i} d y^{j} \\
& +b^{2}(t, y) d y^{2}
\end{aligned}
$$

The nonzero components of Einstein tensor can be written as $[47,52]$

$$
\begin{aligned}
G_{00}= & 3\left[\frac{\dot{a}}{a}(\dot{a} a+\dot{b} b)-\frac{n^{2}}{b^{2}}\left(\frac{a^{\prime \prime}}{a} \frac{a^{\prime}}{a}\left(\frac{a^{\prime}}{a}-\frac{b^{\prime}}{b}\right)\right)+k \frac{n^{2}}{b^{2}}\right], \\
G_{i j}= & \frac{a^{2}}{b^{2}} \gamma_{i j} \\
& \times\left[\frac{a^{\prime}}{a}\left(\frac{a^{\prime}}{a}+2 \frac{n^{\prime}}{n}\right)-\frac{b^{\prime}}{b}\left(\frac{n^{\prime}}{n}+2 \frac{a^{\prime}}{a}\right)+2 \frac{a^{\prime \prime}}{a}+\frac{n^{\prime \prime}}{n}\right] \\
& +\frac{a^{2}}{n^{2}} \gamma_{i j}\left[\frac{\dot{a}}{a}\left(-\frac{\dot{a}}{a}+2 \frac{\dot{n}}{n}\right)-2 \frac{\ddot{a}}{a}+\frac{\dot{b}}{b}\left(-2 \frac{\dot{a}}{a}+\frac{\dot{n}}{n}\right)-\frac{\ddot{b}}{b}\right] \\
& -k \gamma_{i j},
\end{aligned}
$$




$$
\begin{gathered}
G_{05}=3\left(\frac{n^{\prime}}{n} \frac{\dot{a}}{a}+\frac{a^{\prime}}{a} \frac{\dot{b}}{b}-\frac{\dot{a}^{\prime}}{a}\right), \\
G_{55}=3\left[\frac{a^{\prime}}{a}\left(\frac{a^{\prime}}{a}+\frac{n^{\prime}}{n}\right)-\frac{b^{2}}{n^{2}}\left(\frac{\dot{a}}{a}\left(\frac{\dot{a}}{a}-\frac{\dot{n}}{n}\right)+\frac{\ddot{a}}{a}\right)-k \frac{b^{2}}{a^{2}}\right],
\end{gathered}
$$

where $k$ denotes the curvature of space $k=0,+1,-1$ for flat, closed, and open universe, respectively, and $\gamma_{i j}$ is the metric for the maximally symmetric three-dimensional space. The primes and dots denote the derivatives with respect to $y$ and $t$, respectively. The three-dimensional brane is assumed at $y=$ 0 . The Einstein equations are $G_{\mu \nu}=\kappa_{5}^{2} T_{\mu \nu}$, where the stressenergy momentum tensor has bulk and brane components and can be written as $[47,52]$

$$
T_{\nu}^{\mu}=\left.T_{\nu}^{\mu}\right|_{\sigma, b}+\left.T_{\nu}^{\mu}\right|_{m, b}+\left.T_{\nu}^{\mu}\right|_{\Lambda, B}+\left.T_{\nu}^{\mu}\right|_{m, B}
$$

where

$$
\begin{gathered}
\left.T_{\nu}^{\mu}\right|_{\sigma, b}=\frac{\delta(y)}{b} \operatorname{diag}(-\sigma,-\sigma,-\sigma,-\sigma, 0), \\
\left.T_{\nu}^{\mu}\right|_{\Lambda, B}=\operatorname{diag}\left(-\Lambda_{5},-\Lambda_{5},-\Lambda_{5},-\Lambda_{5},-\Lambda_{5}\right), \\
\left.T_{\nu}^{\mu}\right|_{m, b}=\frac{\delta(y)}{b} \operatorname{diag}(-\rho, p, p, p, 0),
\end{gathered}
$$

where $p$ and $\rho$ are the pressure and density on the brane, respectively. Integrating (2) with respect to $y$ around $y=0$ gives the following jump conditions:

$$
\begin{gathered}
a_{+}^{\prime}=-a_{-}^{\prime}=-\frac{\kappa_{5}^{2}}{6} a_{0} b_{0}(\sigma+\rho), \\
n_{+}^{\prime}=-n_{-}^{\prime}=\frac{\kappa_{5}^{2}}{6} b_{0} n_{0}(-\sigma+2 \rho+3 p) .
\end{gathered}
$$

Using (9), one can derive

$$
\begin{gathered}
\dot{\rho}+3 \frac{\dot{a}_{0}}{a_{0}}(\rho+p)=-\frac{2 n_{0}^{2}}{b_{0}} T_{5}^{0}, \\
\frac{1}{n_{0}^{2}}\left[\frac{\ddot{a}_{0}}{a_{0}}+\left(\frac{\dot{a}_{0}}{a_{0}}\right)^{2}-\frac{\dot{a}_{0} \dot{n}_{0}}{a_{0} n_{0}}\right]+\frac{k}{a_{0}^{2}} \\
=\frac{\kappa_{5}^{2}}{3}\left(\Lambda_{5}+\frac{\kappa_{5}^{2} \sigma^{2}}{6}\right) \\
-\frac{\kappa_{5}^{4}}{36}[\sigma(3 p-\rho)+\rho(3 p+\rho)]-\frac{\kappa_{5}^{2}}{3} T_{5}^{5},
\end{gathered}
$$

where $T_{05}$ and $T_{55}$ are the 05 and 55 components of $\left.T_{\mu \nu}\right|_{m, b}$ evaluated on brane. Assuming that the bulk matter relative to bulk vacuum energy is much less than the ratio of the brane matter to the brane vacuum energy, we can neglect the $T_{5}^{5}$ term and this can lead to the derivation of a solution that is largely independent of the bulk dynamics. Considering this approximation and concentrating on the low-energy region with $\rho / \sigma \ll 1,(10)$ can be simplified into

$$
\begin{gathered}
\dot{\rho}+3 H(1+w) \rho=-2 T_{5}^{0}=T, \\
H^{2}=\frac{8 \pi G_{4}}{3}(\rho+\chi)-\frac{k}{a^{2}}+\lambda, \\
\dot{\chi}+4 H \chi \approx 2 T_{5}^{0}=-T .
\end{gathered}
$$

The auxiliary field $\chi$ incorporates nontrivial contributions of dark energy which differ from the standard matter fields confined to the brane. Hence, with the energy exchange $T$ between the bulk and brane, the usual energy conservation is broken down.

\section{RDE in the Bulk-Brane Interaction}

In the present work, we are going to discuss Ricci dark energy (RDE) in bulk-brane interaction. The bulk-brane interaction has been studied for various aspects in the works of [47, 52, 53]. We will denote the energy density of $\operatorname{RDE}$ by $\rho_{\Lambda}$. Since we will consider two dark components in the universe, namely, dark matter and dark energy, we will have $\rho=\rho_{\Lambda}+\rho_{m}$. We assume that the adiabatic equation for the dark matter is satisfied, while it is violated for the dark energy due to the energy exchange between the brane and the bulk $[47,52]$ :

$$
\begin{gathered}
\dot{\rho}_{m}+3 H \rho_{m}=0, \\
\dot{\rho}_{\lambda}+3 H\left(1+\omega_{\Lambda}\right) \rho_{\Lambda}=T .
\end{gathered}
$$

The interaction between bulk and brane is given by the quantity $T=\Gamma \rho_{\Lambda}$, where $\Gamma$ is the rate of interaction. Considering $u=\chi /\left(\rho_{\Lambda}+\rho_{m}\right)$, the above equations lead to [47]

$$
\dot{u}=\left(\frac{3 H u \Omega_{\Lambda}}{\Omega_{\Lambda}+\Omega_{m}}\right)\left[\omega_{\Lambda}-\frac{1}{3}\left(\frac{\Omega_{m}}{\Omega_{\Lambda}}+1\right)-\frac{1+u}{u} \frac{\Gamma}{3 H}\right] .
$$

In our present work, we take $\lambda=0$ and $k=0$. Furthermore, following [47] we have chosen

$$
\Gamma=3 b^{2}(1+u) H .
$$

In flat $(k=0)$ FRW universe, the density of the RDE is given by [54]

$$
\rho_{\Lambda}=3 c^{2}\left(\dot{H}+2 H^{2}\right) .
$$

Differentiating equation (12) with respect to $t$ and using (11) to (13) one has (considering $8 \pi G_{4}=1$ ) for flat universe, that is, $k=0$,

$$
\dot{H}=-\frac{1}{6}\left[3 \rho_{\Lambda}\left(1+\omega_{\Lambda}\right)+3 \rho_{m}+4 \chi\right] .
$$

Using (18) and (12) in (17) we get

$$
\omega_{\Lambda}=\frac{1}{3}\left(1+\frac{\Omega_{m}}{\Omega_{\Lambda}}\right)-\frac{2}{3 c^{2}},
$$




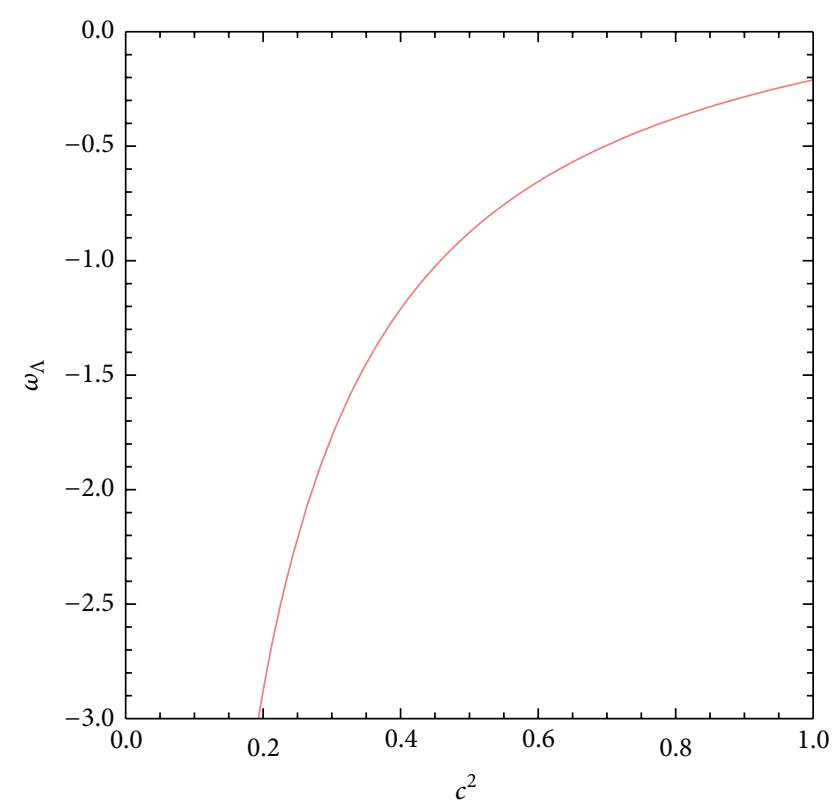

FIGURE 1: Variation of $\omega_{\Lambda}$ with $c^{2}$ pertaining to RDE. We have taken $\Omega_{\Lambda 0}=0.73$ and $\Omega_{m 0}=0.27$.

where we defined

$$
\Omega_{\Lambda}=\frac{\rho_{\Lambda}}{3 H^{2}}, \quad \Omega_{m}=\frac{\rho_{m}}{3 H^{2}}, \quad \Omega_{\chi}=\frac{\chi}{3 H^{2}}
$$

and subsequently

$$
u=\frac{1-\Omega_{\Lambda}-\Omega_{m}}{\Omega_{\Lambda}} .
$$

Using $\Omega_{m 0}=0.27$ and $\Omega_{\Lambda 0}=0.73$ we plot the $\omega_{\Lambda}$ in Figure 1 . In this figure we observe that the EoS parameter is evolving from $<-1$ to $>-1$ as we are changing the value of $c^{2}$. This implies a transition from quintessence to phantom. Moreover, we find that for $c^{2}<0.5$ the EoS is staying below -1 and for $c^{2}>0.5$ the EoS is staying above -1 . In our calculation, we have taken $\Gamma=3 b^{2}(1+u) H$. Furthermore, we have considered scale factor in power law form.

From (19) we can write

$$
\dot{u}=-\left(\frac{3 H u \Omega_{\Lambda}}{\Omega_{\Lambda}+\Omega_{m}}\right)\left[\frac{2}{3 c^{2}}+\frac{b^{2}(1+u)^{2}}{u}\right] .
$$

Since we have $\Omega_{\Lambda}+\Omega_{m}=(1+u)^{-1}$, (22) can be rewritten as

$$
\dot{u}=\frac{3 H u(1+u)}{\Omega_{\Lambda}}\left[\frac{2}{3 c^{2}}+\frac{b^{2}(1+u)^{2}}{u}\right] .
$$

Let us select the scale factor $a$ in the power law form; that is, $a(t)=a_{0} t^{n}$. Then

$$
H=\frac{n}{t} .
$$

Subsequently, (23) becomes

$$
\dot{u}=-\frac{3 n^{2} u(1+u)}{t c^{2}(2 n-1)}\left[\frac{2}{3 c^{2}}+\frac{b^{2}(1+u)^{2}}{u}\right] .
$$

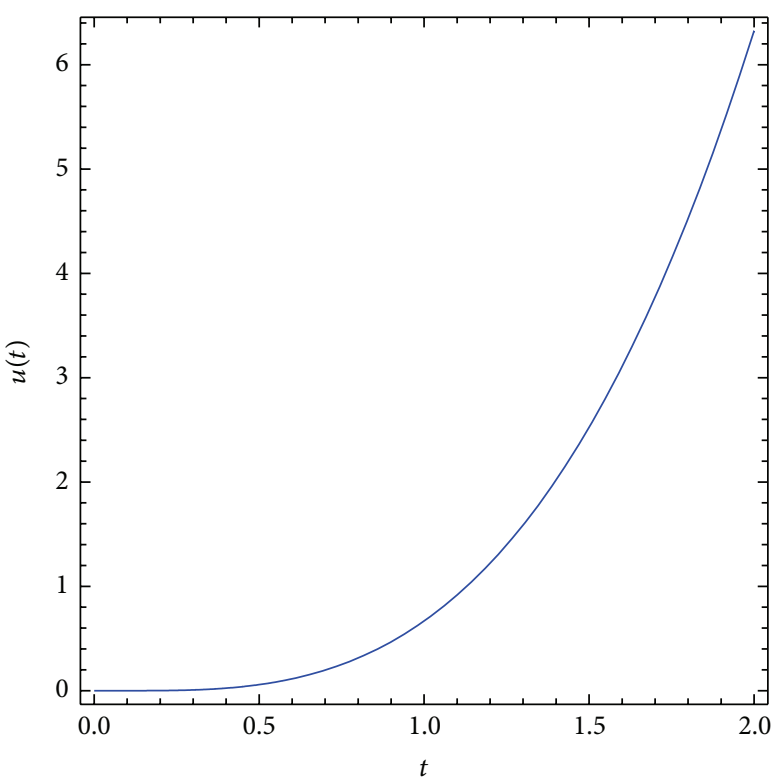

FIGURE 2: Pattern of $u$ with evolution of the universe under power law expansion. We have taken $c=0.7, n=1 / 3$, and $b=2$.

In Figure 2 we have plotted $u$ against $t$. We observe that $u$ is increasing with time. This indicates that nontrivial contributions of dark energy which differ from the standard matter fields confined to the brane are increasing with the evolution of the universe.

\section{Conclusion}

In this work we have investigated the effects of the interaction between a brane universe and the bulk in which it is embedded. Considering the effects of the interaction between a brane universe and the bulk we have obtained the equation of state for the interacting holographic Ricci dark energy density $\rho_{\Lambda}=3 c^{2}\left(\dot{H}+2 H^{2}\right)$ in the flat universe. We have seen that there is a crossing of the barrier $\omega_{\Lambda}=-1$ as $c^{2}$ shifts from $<0.5$ to $>0.5$. If $c^{2}<0.5$, the EoS behaves like phantom $(\leq-1)$ and if $c^{2}>0.5$, the EoS behaves like quintessence $(\geq-1)$. Next we have chosen a particular form of scale factor $a(t)=a_{0} t^{n}$. We expressed $\dot{u}$ as a function of $t$ and solving it numerically we plotted $u$ against $t$ and we found an increasing behavior in $u$. We observed that nontrivial contributions of dark energy which differ from the standard matter fields confined to the brane are increasing with the evolution of the universe.

\section{Acknowledgment}

The author wishes to acknowledge the financial support from the Department of Science and Technology, Government of India, under the Fast Track Scheme for Young Scientists. The Grant no. is SR/FTP/PS-167/2011. 


\section{References}

[1] P. J. E. Peebles and B. Ratra, "The cosmological constant and dark energy," Reviews of Modern Physics, vol. 75, no. 2, pp. 559606, 2003.

[2] E. J. Copeland, M. Sami, and S. Tsujikawa, "Dynamics of dark energy," International Journal of Modern Physics D, vol. 15, no. 11, pp. 1753-1935, 2006.

[3] T. Padmanabhan, "Dark energy: the cosmological challenge of the millennium," Current Science, vol. 88, no. 7, pp. 1057-1067, 2005.

[4] T. Padmanabhan, "Dark energy and gravity," General Relativity and Gravitation, vol. 40, no. 2-3, pp. 529-564, 2008.

[5] L. Amendola and S. Tsujikawa, Dark Energy: Theory and Observations, Cambridge University Press, Cambridge, UK, 2010.

[6] K. Bamba, S. Capozziello, S. I. Nojiri, and S. D. Odintsov, "Dark energy cosmology: the equivalent description via different theoretical models and cosmography tests," Astrophysics and Space Science, vol. 342, no. 1, pp. 155-228, 2012.

[7] J. Yoo and Y. Watanabe, "Theoretical models of dark energy," International Journal of Modern Physics D, vol. 21, Article ID 1230002, 53 pages, 2012.

[8] M. Trodden, "Dark energy and cosmology," 2012, http://arxiv .org/abs/1212.6399.

[9] L. Marek-Crnjac, M. Naschie, and J. He, "Chaotic fractals at the root of relativistic quantum physics and cosmology," International Journal of Modern Nonlinear Theory and Application, vol. 2, pp. 78-88, 2013.

[10] M. El Naschie, "Dark energy from Kaluza-Klein spacetime and Noether's theorem via lagrangian multiplier method," Journal of Modern Physics, vol. 4, pp. 757-760, 2013.

[11] M. Naschie, "Quantum entanglement: where dark energy and negative gravity plus accelerated expansion of the universe comes from," Journal of Quantum Information Science, vol. 3, pp. 57-77, 2013.

[12] S. M. Carroll, M. Hoffman, and M. Trodden, "Can the dark energy equation-of-state parameter w be less than -1 ?" Physical Review D, vol. 68, Article ID 023509, 11 pages, 2003.

[13] A. Melchiorri, L. Mersini, C. J. Odman, and M. Trodden, "The state of the dark energy equation of state," Physical Review D, vol. 68, Article ID 043509, 7 pages, 2003.

[14] E. N. Saridakis, "Theoretical limits on the equation-of-state parameter of phantom cosmology," 2008, http://arxiv.org/ abs/0811.1333.

[15] Y.-F. Cai, E. N. Saridakis, M. R. Setare, and J.-Q. Xia, "Quintom cosmology: theoretical implications and observations," Physics Reports, vol. 493, no. 1, pp. 1-60, 2010.

[16] T. Padmanabhan, "Accelerated expansion of the universe driven by tachyonic matter," Physical Review D, vol. 66, Article ID 021301, 2002.

[17] R. R. Caldwell, "A phantom menace? Cosmological consequences of a dark energy component with super-negative equation of state," Physics Letters B, vol. 545, no. 1-2, pp. 23-29, 2002.

[18] A. Kamenshchik, U. Moschella, and V. Pasquier, "An alternative to quintessence," Physics Letters B, vol. 511, no. 2-4, pp. 265-268, 2001.

[19] T. Chiba, T. Okabe, and M. Yamaguchi, "Kinetical driven quintessence," Physical Review D, vol. 62, Article ID 023511, 2000 .
[20] H. Wei, R.-G. Cai, and D.-F. Zeng, "Hessence: a new view of quintom dark energy," Classical and Quantum Gravity, vol. 22, no. 16, pp. 3189-3202, 2005.

[21] F. Piazza and S. Tsujikawa, "Dilatonic ghost condensate as dark energy," Journal of Cosmology and Astroparticle Physics, vol. 2004, article 004, 2004.

[22] D. Pavon and W. Zimdahl, "Holographic dark energy and cosmic coincidence," Physics Letters B, vol. 628, pp. 206-210, 2005.

[23] Supernova Search Team Collaboration, "Type Ia supernova discoveries at $z>1$ from the Hubble Space Telescope: evidence for past deceleration and constraints on dark energy evolution," The Astrophysical Journal, vol. 607, no. 2, p. 665, 2004.

[24] Boomerang Collaboration, "A flat Universe from high-resolution maps of the cosmic microwave background radiation," Nature, vol. 404, pp. 955-959, 2000.

[25] SDSS Collaboration, "Detection of the baryon acoustic peak in the large-scale correlation function of SDSS luminous red galaxies," The Astrophysical Journal, vol. 633, no. 2, p. 560, 2005.

[26] R. Bousso, "The holographic principle," Reviews of Modern Physics, vol. 74, no. 3, pp. 825-874, 2002.

[27] M. Li, "A model of holographic dark energy," Physics Letters B, vol. 603, pp. 1-5, 2004.

[28] C.-J. Feng, "Statefinder diagnosis for Ricci dark energy," Physics Letters B, vol. 670, pp. 231-234, 2008.

[29] A. G. Cohen, D. B. Kaplan, and A. E. Nelson, "Effective field theory, black holes, and the cosmological constant," Physical Review Letters, vol. 82, no. 25, pp. 4971-4974, 1999.

[30] S. D. H. Hsu, "Entropy bounds and dark energy," Physics Letters $B$, vol. 594, pp. 13-16, 2004.

[31] C. Gao, F. Wu, X. Chen, and Y.-G. Shen, "Holographic dark energy model from Ricci scalar curvature," Physical Review D, vol. 79, no. 4, Article ID 043511, 7 pages, 2009.

[32] L. Xu, J. Lu, and W. Li, "Generalized holographic and Ricci dark energy models," The European Physical Journal C, vol. 64, no. 1, pp. 89-95, 2009.

[33] R. G. Cai, B. Hu, and Y. Zhang, "Holography, UV/IR relation, causal entropy bound, and dark energy," Communications in Theoretical Physics, vol. 51, no. 5, p. 954, 2009.

[34] C.-J. Feng, "Statefinder diagnosis for Ricci dark energy," Physics Letters B, vol. 670, no. 3, pp. 231-234, 2008.

[35] C.-J. Feng, "Reconstructing quintom from Ricci dark energy," Physics Letters B, vol. 672, pp. 94-97, 2009.

[36] C.-J. Feng, "Reconstructing $f(R)$ theory from Ricci dark energy," Physics Letters B, vol. 676, no. 4-5, pp. 168-172, 2009.

[37] C.-J. Feng and X.-Z. Li, "Viscous Ricci dark energy," Physics Letters B, vol. 680, no. 4, pp. 355-358, 2009.

[38] C.-J. Feng and X. Zhang, "Holographic Ricci dark energy in Randall-Sundrum braneworld: avoidance of big rip and steady state future," Physics Letters B, vol. 680, no. 5, pp. 399-403, 2009.

[39] V. A. Rubakov and M. E. Shaposhnikovubakov, "Extra spacetime dimensions: towards a solution to the cosmological constant problem," Physics Letters B, vol. 125, no. 2-3, pp. 139-143, 1983.

[40] I. Antoniadis, "A possible new dimension at a few TeV," Physics Letters B, vol. 246, no. 3-4, pp. 377-384, 1990.

[41] P. Binetruy, C. Deffayet, U. Ellwanger, and D. Langlois, "Brane cosmological evolution in a bulk with cosmological constant," Physics Letters B, vol. 477, pp. 285-291, 2000. 
[42] C. van de Bruck, M. Dorcab, C. J. A. P. Martins, and M. Parryd, "Cosmological consequences of the brane/bulk interaction," Physics Letters B, vol. 495, no. 1-2, pp. 183-192, 2000.

[43] S. Nojiri, S. D. Odintsov, and S. Ogushi, "FriedmannRobertson-Walker brane cosmological equations from the fivedimensional bulk (A)dS black hole," International Journal of Modern Physics A, vol. 17, no. 32, p. 4809, 2002.

[44] C. Deffayet, "Cosmology on a brane in Minkowski bulk," Physics Letters B, vol. 502, pp. 199-208, 2001.

[45] M. Jamil, K. Karami, and A. Sheykhi, "Restoring new agegraphic dark energy in RS II braneworld," International Journal of Theoretical Physics, vol. 50, no. 10, pp. 3069-3077, 2011.

[46] E. N. Saridakis, "Restoring holographic dark energy in brane cosmology," Physics Letters B, vol. 660, no. 3, pp. 138-143, 2008.

[47] M. R. Setare, "Bulk-brane interaction and holographic dark energy," Physics Letters B, vol. 642, no. 5-6, pp. 421-425, 2006.

[48] A. Sheykhi, "Brane-Bulk energy exchange and agegraphic dark energy," International Journal of Modern Physics D, vol. 19, no. 3, p. 305, 2010.

[49] Y. S. Myung and J. Y. Kim, "Brane-bulk interaction and holographic principle," Classical and Quantum Gravity, vol. 20, no. 11, pp. L169-L175, 2003.

[50] K. Y. Kim, H. W. Lee, and Y. S. Myung, "Holographic interacting dark energy in the braneworld cosmology," Modern Physics Letters A, vol. 22, no. 35, pp. 2631-2645, 2007.

[51] E. N. Saridakis, "Holographic dark energy in braneworld models with moving branes and the $w=-1$ crossing," Journal of Cosmology and Astroparticle Physics, vol. 2008, article 020, 2008.

[52] R.-G. Cai, Y. Gong, and B. Wang, "Super-acceleration on the brane through energy flow from the bulk," Journal of Cosmology and Astroparticle Physics, vol. 2006, article 006, 2006.

[53] M. R. Setare and E. N. Saridakis, "Braneworld models with a non-minimally coupled phantom bulk field: a simple way to obtain the -1-crossing at late times," Journal of Cosmology and Astroparticle Physics, vol. 2009, article 002, 2009.

[54] C.-J. Feng, "Statefinder diagnosis for Riccidark energy," Physics Letters B, vol. 670, no. 3, pp. 231-234, 2008. 

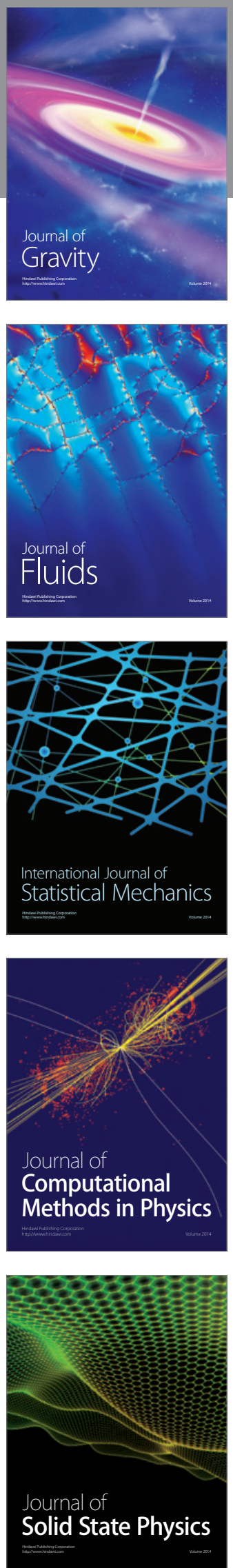

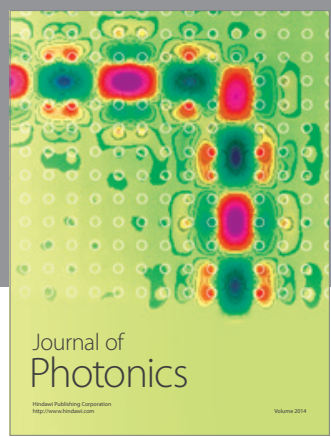

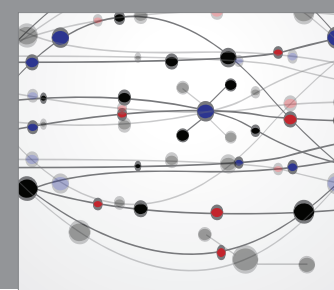

The Scientific World Journal

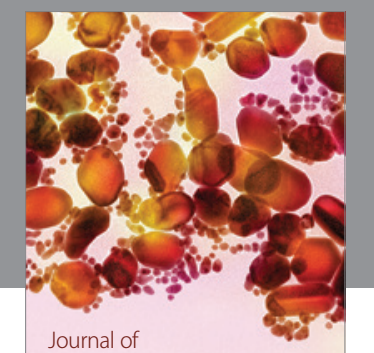

Soft Matter
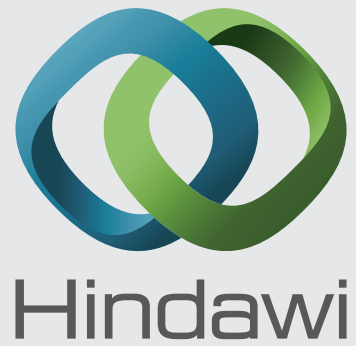

Submit your manuscripts at

http://www.hindawi.com
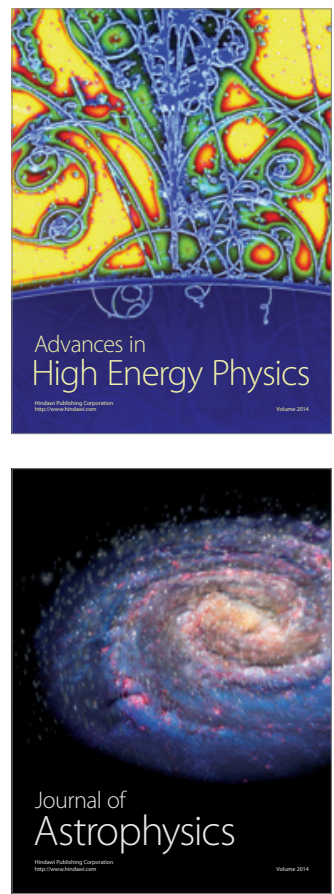
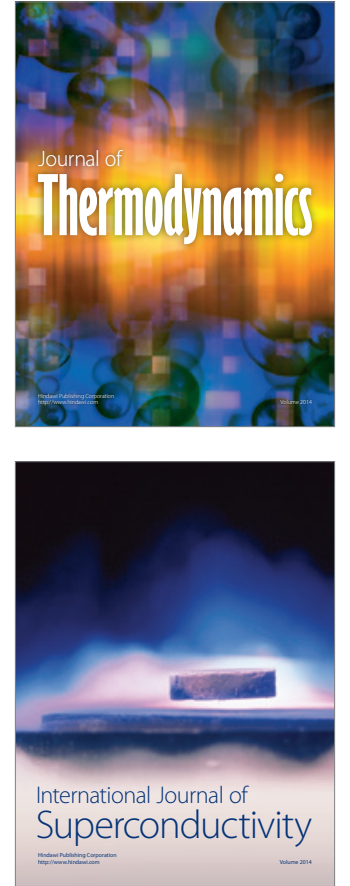
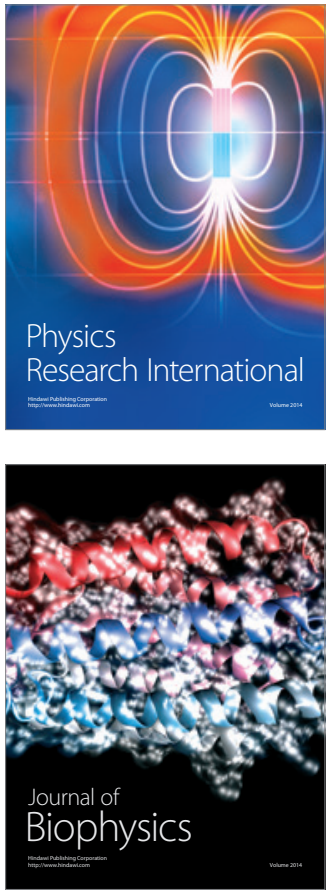
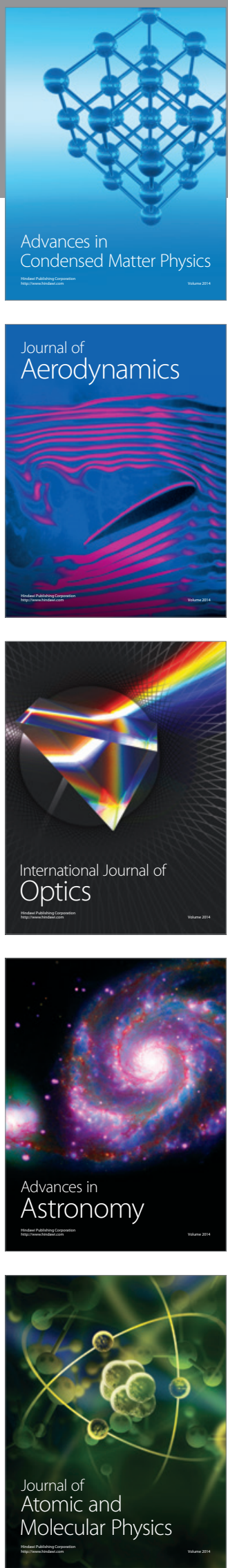